\title{
The high energy gamma-ray emission expected from Tycho's supernova remnant
}

\author{
H. J. Völk ${ }^{1}$, E. G. Berezhko ${ }^{2}$, L. T. Ksenofontov ${ }^{2}$, and G. P. Rowell ${ }^{1}$ \\ 1 Max Planck Institut für Kernphysik, Postfach 103980, 69029 Heidelberg, Germany \\ e-mail: Heinrich.Voelk@mpi-hd.mpg.de; Gavin.Rowell@mpi-hd.mpg.de \\ 2 Institute of Cosmophysical Research and Aeronomy, 31 Lenin Ave., 677891 Yakutsk, Russia \\ e-mail: berezhko@ikfia.ysn.ru; ksenofon@ikfia.ysn.ru
}

Received 19 April 2002 / Accepted 24 September 2002

\begin{abstract}
A nonlinear kinetic model of cosmic ray (CR) acceleration in supernova remnants (SNRs) is used to describe the properties of Tycho's SNR. Observations of the expansion characteristics and of the nonthermal radio and X-ray emission spectra, assumed to be of synchrotron origin, are used to constrain the overall dynamical evolution and the particle acceleration parameters of the system, in addition to what is known from independent estimates of the distance and thermal X-ray observations. It is shown that a very efficient production of nuclear cosmic rays, leading to strong shock modification, and a large downstream magnetic field strength $B_{\mathrm{d}} \approx 240 \mu \mathrm{G}$ are required to reproduce the observed synchrotron emission from radio to X-ray frequencies. This field strength is still well within the upper bound for the effective magnetic field, consistent with the acceleration process. The $\pi^{0}$-decay $\gamma$-ray flux turns out to be somewhat greater than the inverse Compton (IC) flux off the Cosmic Microwave Background at energies below $1 \mathrm{TeV}$, dominating it strongly at $10 \mathrm{TeV}$. The predicted $\mathrm{TeV} \gamma$-ray flux is consistent with but close to the very low upper limit recently obtained by HEGRA. A future detection at $\epsilon_{\gamma} \sim 10 \mathrm{TeV}$ would clearly indicate hadronic emission.
\end{abstract}

Key words. acceleration of particles - radiation mechanisms: non-thermal - stars: supernovae: individual: Tycho's SNR radio continuum: ISM - X-rays: ISM - gamma rays: theory

\section{Introduction}

The observations of Tycho's supernova remnant (SNR) with the HEGRA stereoscopic system of imaging atmospheric Cherenkov telescopes (IACTs) on La Palma have recently been analyzed. The object has long been considered as a prototype candidate hadronic CR source in the Northern Hemisphere (e.g. Drury et al. 1994), although it was always clear that the sensitivity of the present generation of IACTs is marginal for a detection. In fact, after $\sim 65$ hours of observation time, HEGRA did not detect Tycho's SNR, but it could establish a very low $3 \sigma$ upper flux limit of $5.78 \times 10^{-13}$ photons $\mathrm{cm}^{-2} \mathrm{~s}^{-1}$, or 33 milli-Crab, at energies $>1 \mathrm{TeV}$ (Aharonian et al. 2001). This value is about a factor 4 lower than the one previously published by the Whipple collaboration (Buckley et al. 1998), assuming a spectral index of -1.1 for the comparison. In the above HEGRA paper on Tycho the existing radio and X-ray synchrotron observations were used to infer a lower limit to the mean magnetic field strength in the remnant due to the nondetection of Inverse Compton (IC) emission off the Cosmic Microwave Background (CMB). At the same time, published estimates of the hadronic $\pi^{0}$-decay gamma-ray

Send offprint requests to: H. J. Völk,

e-mail: Heinrich.Voelk@mpi-hd.mpg.de emission (Berezhko \& Völk 1997) were employed and scaled to the parameters of Tycho's SNR, to compare with the new upper flux limit. The predictions from the time-dependent kinetic model (Berezhko et al. 1996) were also renormalized to take the expected deviations from spherical symmetry of the nucleon injection rate for a Type Ia SNR into account, and were found to be consistent with the present HEGRA nondetection in $\gamma$-rays, although the predicted flux values for the hadronic emission were only slightly smaller than the observational upper limit.

This tantalizing situation has prompted us to model the acceleration of both electrons and protons in detail with the nonlinear kinetic theory, using the observed synchrotron emission as a constraint on the electron acceleration characteristics, and thereby to model the hadronic and IC $\gamma$-ray emission simultaneously.

We demonstrate that, together with the renormalization, the existing data are consistent with very efficient acceleration of CR nuclei at the SN shock wave which converts a significant fraction of the initial SNR energy content into CR energy. This energy is distributed between energetic protons and electrons in a proportion similar to that of the Galactic CRs. Therefore Tycho's SNR might indeed be a typical Galactic CR source. Direct evidence for the inferred strong production of 
nucleonic CRs in Tycho would not only require a $\gamma$-ray detection as such, but in particular a spectrum measurement, ideally beginning at photon energies of about $100 \mathrm{MeV}$ up to the highest energies. Of particular importance would be measurements in excess of $10 \mathrm{TeV}$ particle energy which are beyond the reach of the accelerated electron component that suffers a cutoff at energy of about $50 \mathrm{TeV}$ due to synchrotron losses in our model. This specific spectral behavior implies a high magnetic field strength of about $40 \mu \mathrm{G}$ upstream of the outer SNR shock. Such a field strength is indeed required to fit the nonthermal radio and $\mathrm{X}$-ray synchrotron emission. We shall give an upper bound on the effective magnetic field in the discussion section. It well encompasses the deduced field strength.

\section{Model}

A supernova (SN) explosion ejects a shell of matter with total energy $E_{\mathrm{sn}}$ and mass $M_{\mathrm{ej}}$. During an initial period the shell material has a broad distribution in velocity $v$. The fastest part of these ejecta is described by a power law $\mathrm{d} M_{\mathrm{ej}} / \mathrm{d} v \propto v^{2-k}$ (e.g. Jones et al. 1981; Chevalier 1982). The interaction of the ejecta with the interstellar medium (ISM) there creates a strong shock which accelerates particles.

Our nonlinear model (Berezhko et al. 1996; Berezhko \& Völk 1997) is based on a fully time-dependent solution of the CR transport equations together with the gas dynamic equations in spherical symmetry. Regarding the coefficients in the transport equations and the boundary conditions as well as the approximation of spherical symmetry, we shall introduce a number of approximations as follows (see also the discussion in Berezhko et al. 2002):

The CR diffusion coefficient is taken as the Bohm limit

$\kappa(p)=\kappa(m c)(p / m c)$,

where $\kappa(m c)=m c^{2} /(3 e B), e$ and $m$ are the particle charge and mass, $p$ denotes the particle momentum, $B$ is the magnetic field strength, and $c$ is the speed of light. This limiting value is consistent with the high, probably turbulently amplified magnetic field strength (Lucek \& Bell 2000) due to the strong pressure gradient of the energetic particles in the very strong shock of the young remnant. In fact, Eq. (1) uses the relativistic form of Bohm diffusion, equal for both electrons and nuclei. In fact, the actual form of $\kappa(p)$ at low momenta $p \ll p_{\max }$ does not influence the particle spectrum if $k(p)$ is a strongly increasing function of $p$. In addition at nonrelativistic proton energies $p<m_{\mathrm{p}} c$, even if the electron and proton diffusion coefficients are different at the same $p$, their spectra $f(p)$ are essentially the same because these low energy particles do not produce any shock modification and therefore their spectrum is $f(p) \propto p^{-q_{\mathrm{s}}}$, where $q_{\mathrm{s}}$ is the spectral index appropriate for the subshock compression ratio $\sigma_{\mathrm{s}}$, calculated below.

The number of suprathermal protons injected into the acceleration process is described by a dimensionless injection parameter $\eta$ which is a fixed fraction of the ISM particles entering the shock front. For simplicity it is assumed that the injected particles have a velocity four times higher than the postshock sound speed. It is expected that ion injection is quite efficient at the quasi-parallel portion of the shock surface, where it is characterized by the value $\eta \sim 10^{-4}$ (see Völk et al. 2002 for details). In momentum space the accelerated (nonthermal) proton distribution function grows smoothly out of the downstream thermal distribution (e.g. Malkov \& Völk 1995).

Assuming nucleon injection to occur through the leakage of suprathermal particles into the upstream region from behind the shock, the relevant injection velocity parallel to the magnetic field is $V_{\mathrm{s}} \cos ^{-1} \phi$, where $V_{\mathrm{s}}$ denotes the (radial) shock velocity and $\phi$ is the angle between the shock normal and the downstream magnetic field direction; it is refracted away from the shock normal. Therefore the injection is expected to be strongly suppressed at the quasi-perpendicular surface fraction of the shock and this surface fraction is larger than that of the quasi-parallel fraction due to the field refraction. This lack of symmetry in the actual SNR can be approximately taken into account by a renormalization factor $f_{\mathrm{re}}<1 / 2$ which diminishes the nucleonic $\mathrm{CR}$ production efficiency as calculated in the spherical model, and all effects associated with it. A more detailed estimate, taking also the diffusive broadening of the quasi-parallel area into account, yields roughly $f_{\text {re }}=0.15$ to 0.25 (Völk et al. 2002).

This is not necessarily in conflict with the radio polarization result of Reynoso et al. (1997) and Dickel et al. (1991) who obtain from their VLA measurements a roughly radial ordered magnetic field component over most of the shock surface. The fractional polarization of $10 \%$ to $15 \%$ is rather small (Dickel et al. 1991), indicating a rather small excess of ordered radial magnetic field (see also the discussion in Reynolds \& Gilmore 1993). Obviously Tycho's SNR is not a magnetic monopole. To resolve this puzzle we argue that there exist at least two physical processes which tend to produce radial field directions in a remnant that is still in the sweep-up phase and as a whole embeded in a uniform ISM and magnetic field. First of all, such young SNRs as Tycho's are Rayleigh-Taylor unstable during the sweep-up phase (Gull 1973; Velázquez et al. 1998). Secondly, the ejecta themselves may be so nonuniform that they can wrinkle the blast wave surface (Aschenbach et al. 1995). In addition, the ambient ISM around Tycho appears to be unexpectedly nonuniform as well, cf. Reynoso et al. (1997). Even though these irregularities are of a macroscopic, hydromagnetic nature, their size spectrum probably extends to quite small scales. The acceleration scales can even be much smaller. In fact, the acceleration scales of protons with multi-GeV energies, on whose strong self-excited magnetic irregularities (Bell 1978) the electrons may parasitically accelerate as well, is of order $10^{-4}$ times the shock radius or even smaller, and the proton scales are connected with quasi-parallel, i.e. quasi-radial field line portions. On other portions no acceleration occurs, and they will not be illuminated by synchrotron emitting electrons. Thus it is possible that the magnetic field appears essentially radial over the remnant surface even to the VLA. We leave it open here to which extent small-scale magnetic irregularities that lead to measurable polarization effects can also be nonlinearly produced by the accelerated particle population itself.

Due to their small mass $m_{\mathrm{e}}$, suprathermal electrons cannot resonantly interact with the hydromagnetic wave 
turbulence created by the energetically dominant accelerating ions. Therefore electrons can only participate in the diffusive shock acceleration process at high energies $\leq m_{\mathrm{e}} c^{2}$, albeit still nonrelativistic as far as nuclei are concerned. Presumably they are injected by electrostatic fluctuations (e.g. Malkov \& Drury 2001). For simplicity we can consider their acceleration to start from the same momentum as protons start, if we restrict our further consideration to relativistic electrons, at energies possibly far above their actual injection energy. What counts alone for the considerations in this paper is that at energies corresponding to those of relativistic protons - only those energies play a role for the observed synchrotron emission - electrons have exactly the same dynamics as the protons. Therefore, below the momenta where synchrotron losses become important, the electron distribution function has at any given time the form

$f_{\mathrm{e}}(p)=K_{\mathrm{ep}} f(p)$

with a factor $K_{\mathrm{ep}}$ that is of the order of $10^{-2}$ for the average CRs in the Galaxy.

Clearly, from the point of view of injection/acceleration theory, we must treat $K_{\text {ep }}$ together with $B$ as free parameters, and $\eta$ as well as $f_{\text {re }}$ as theoretically not yet accurately calculable parameters, to be quantitatively determined by comparison with observations.

The electron distribution function $f_{\mathrm{e}}(p)$ deviates only at sufficiently large momenta from Eq. (2) due to synchrotron losses, which are taken into account by supplementing the ordinary diffusive transport equation by a radiative loss term. The solution of the dynamic equations at each instant of time yields the CR spectrum and the spatial distributions of CRs and thermal gas. This allows the calculation of the expected flux $F_{\gamma}^{\pi}\left(\epsilon_{\gamma}\right)$ of $\gamma$-rays from $\pi^{0}$-decay due to $p-p$ collisions of CRs with the gas nuclei. The choice of $K_{\text {ep }}$ allows one then to determine the electron distribution function in the energy region where losses can be neglected, and to calculate the associated emission. Details about this calculation are given in Berezhko et al. (2002).

\section{Results and discussion}

Tycho was a type Ia SN. Therefore we use typical SN Ia parameters in our calculations: ejected mass $M_{\mathrm{ej}}=1.4 M_{\odot}$, $k=7$, and a uniform ambient ISM with hydrogen number density $N_{\mathrm{H}}=0.5 \mathrm{~cm}^{-3}$ and temperature $T_{0}=10^{4} \mathrm{~K}$. Following Chevalier et al. (1980) (see also Heavens 1984; Reynolds \& Ellison 1992; Aharonian et al. 2001) we in addition adopt a distance $d=2.3 \mathrm{kpc}$, and a present radius of $\sim 4^{\prime}$. These parameters are similar to those inferred by Dickel \& Jones (1985) and Smith et al. (1988). Note that SNR and CR dynamics are not sensitive to the precise value used for $T_{0}$, because the shock structure is mainly determined by the Alfvénic Mach number.

We use an upstream magnetic field value $B_{0}=40 \mu \mathrm{G}$, which is required to provide the required shape of the synchrotron spectrum in the radio and X-ray bands (see below).

The gas dynamic problem is characterized by the following length, time, and velocity scales:

$R_{0}=\left(3 M_{\mathrm{ej}} / 4 \pi \rho_{0}\right)^{1 / 3}, t_{0}=R_{0} / V_{0}, V_{0}=\sqrt{2 E_{\mathrm{sn}} / M_{\mathrm{ej}}}$, which are the sweep-up radius, sweep-up time and mean ejecta speed respectively. Here $\rho_{0}=1.4 m_{\mathrm{p}} N_{\mathrm{H}}$ is the ISM mass density, $m_{\mathrm{p}}$ is the proton mass.

According to Chevalier (1982), an analytical approximation to the shock expansion law during the free expansion phase $\left(t<t_{0}\right)$ is then

$R_{\mathrm{s}} \propto E_{\mathrm{sn}}^{(k-3) / 2 k} \rho_{0}^{-1 / k} t^{(k-3) /(k-2)}$,

which for $k=7$ gives

$R_{\mathrm{s}} \propto\left(E_{\mathrm{sn}}^{2} / \rho_{0}\right)^{1 / 7} t^{4 / 5}$.

In the adiabatic phase $\left(t \gtrsim t_{0}\right)$ we have

$R_{\mathrm{S}} \propto\left(E_{\mathrm{sn}} / \rho_{0}\right)^{1 / 5} t^{2 / 5}$.

The observed mean expansion law of Tycho's SNR (Tan \& Gull $1985)$ is $R_{\mathrm{s}} \propto t^{\mu}$ with $\mu=0.46 \pm 0.02$. This is consistent with the average over the outer rim of the remnant, as obtained by Reynoso et al. (1997), even though the latter authors found significant azimuthal variations of the expansion rate, indicating a substantial nonuniformity of the SNR environment, as mentioned above. On the whole therefore Tycho's SNR should be near the adiabatic phase (Strom et al. 1982; Tan \& Gull 1985; Reynoso et al. 1997).

The calculations together with the azimuthally averaged experimental data are shown in Fig. 1. An explosion energy $E_{\mathrm{sn}}=0.27 \times 10^{51} \mathrm{erg}$ is taken (in addition to the above ISM density, and the ejecta mass) to fit the observed SNR size $R_{\mathrm{S}}$ and its expansion rate $V_{\mathrm{s}}$. This value is again in basic agreement with the earlier determinations referred to above.

According to Fig. 1a Tycho is indeed nearing the adiabatic phase. To fit the observed radio spectral index (see below) we assume a proton injection rate $\eta=3 \times 10^{-4}$. This leads to a significant nonlinear modification of the shock which at the current age of $t=428$ yrs has a total compression ratio $\sigma=5.7$ and a subshock compression ratio $\sigma_{\mathrm{s}}=3.5$ (Fig. 1b).

We should at this point ask the question, how sensitive these results are to the specific choice of the overall parameters. These parameters are partly the result of analyses of the thermal $\mathrm{X}$-ray emission. The corresponding X-ray models need to take into account of non-equilibrium electron temperatures and nonequilibrium ionisation states behind the outer shock as well as nonsolar chemical composition in the ejecta which are in part heated by the reverse shock. As a consequence, different workers do not obtain identical results. The differences to our set of parameters is most pronounced in the results of Hamilton et al. (1986) who derived the different values $E_{\mathrm{sn}}=0.7 \times 10^{51} \mathrm{erg}$, $N_{\mathrm{H}}=0.28 \mathrm{~cm}^{-3}$, and $d=3.1 \mathrm{kpc}$. One way of comparing the consequences of such different inputs into our theory is to check on their internal consistency. Taking into account that the linear size $R_{\mathrm{S}} \propto d$, then it follows from Eq. (4) that $E_{\mathrm{sn}} \propto N_{\mathrm{H}}^{1 / 2} d^{7 / 2}$ for a given source. Changing our parameters $N_{\mathrm{H}}$ and $d$ to $N_{\mathrm{H}}=0.28 \mathrm{~cm}^{-3}$ and $d=3.1 \mathrm{kpc}$, respectively, we obtain $E_{\mathrm{sn}}=0.57 \times 10^{51} \mathrm{erg}$ for the Hamilton et al. case, not too different from their actual value for $E_{\mathrm{sn}}$. We can also discuss the change expected for the resulting $\pi^{0}$-decay $\gamma$-ray integral flux $F_{\gamma}^{\pi}$. Since in the strongly nonlinear case, which is characterized by efficient $\mathrm{CR}$ acceleration, the number density of 


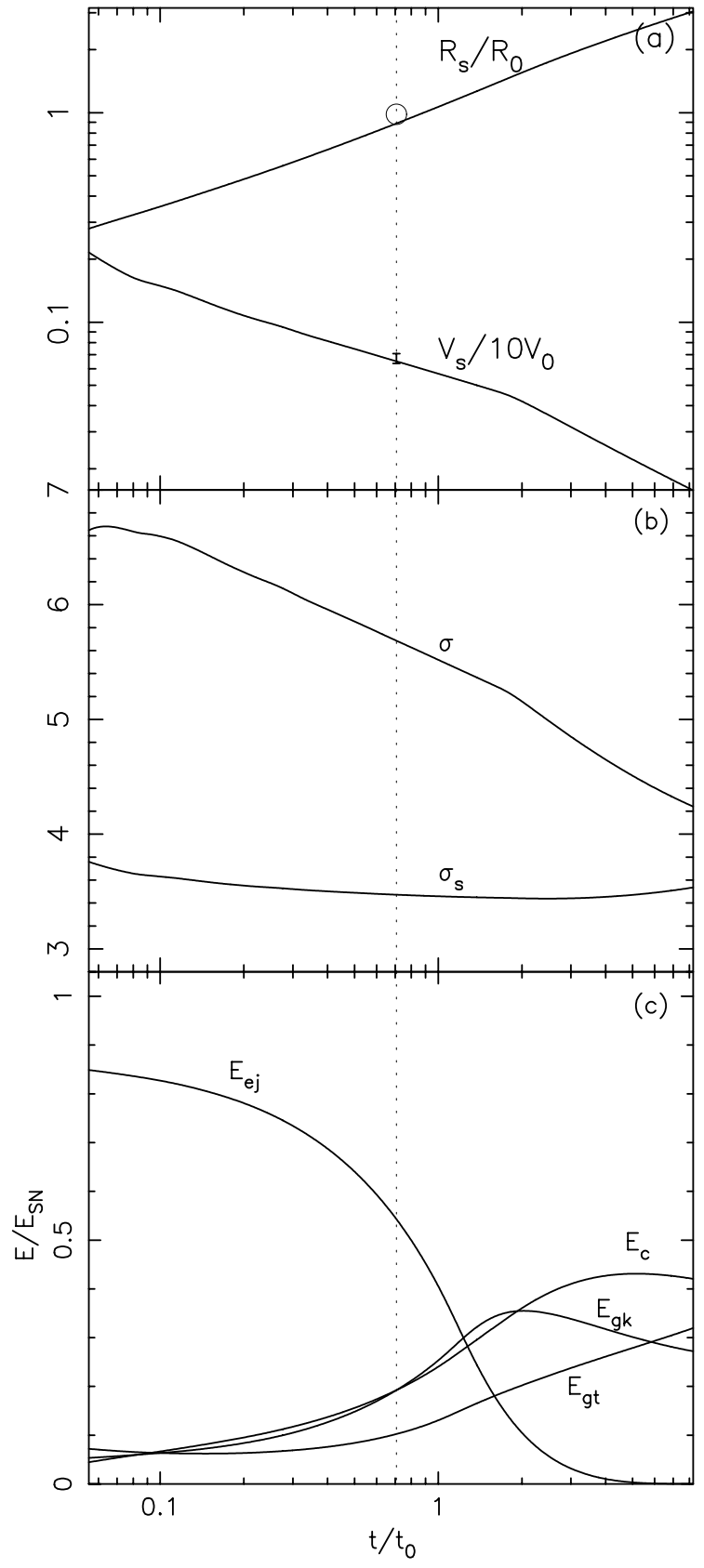

Fig. 1. a) Shock radius $R_{\mathrm{s}}$ and shock speed $V_{\mathrm{s}} ;$ b) total shock $(\sigma)$ and subshock $\left(\sigma_{\mathrm{s}}\right)$ compression ratios; c) ejecta $\left(E_{\mathrm{ej}}\right), \mathrm{CR}\left(E_{\mathrm{c}}\right)$, gas thermal $\left(E_{\mathrm{gt}}\right)$, and gas kinetic $\left(E_{\mathrm{gk}}\right)$ energies as a function of time. Scale values are $R_{0}=2.72 \mathrm{pc}, V_{0}=4402 \mathrm{~km} \mathrm{~s}^{-1}, t_{0}=605$ years. The dotted vertical line marks the current epoch. The observed size and speed of the shock (Tan \& Gull 1985) are shown as well.

accelerated CRs at relativistic energies is proportional to $\rho_{0} V_{\mathrm{s}}^{2}$ (e.g. Berezhko et al. 1996; Berezhko \& Völk 1997), the occupied volume goes like $R_{\mathrm{s}}^{3}$, and the $\pi^{0}$-decay $\gamma$-ray production is proportional to the gas density $\rho_{0}$, we have $F_{\gamma}^{\pi} \propto N_{\mathrm{H}}^{2} R_{\mathrm{s}}^{3} V_{\mathrm{s}}^{2} d^{-2}$. Inserting Hamilton et al.'s values for $d$ and $N_{\mathrm{H}}$, the $\pi^{0}$-decay $\gamma$-ray flux would be decreased to 0.77 times our value given in Fig.6 below. Given our value of the magnetic field, we can do the same for the electron-proton ratio $K_{\mathrm{ep}}$, given the measured radio energy flux $v S_{v}$ (see Fig. 3). The value of $K_{\text {ep }}$ would be lowered by a factor of 1.3 times our value. The IC $\gamma$-ray flux

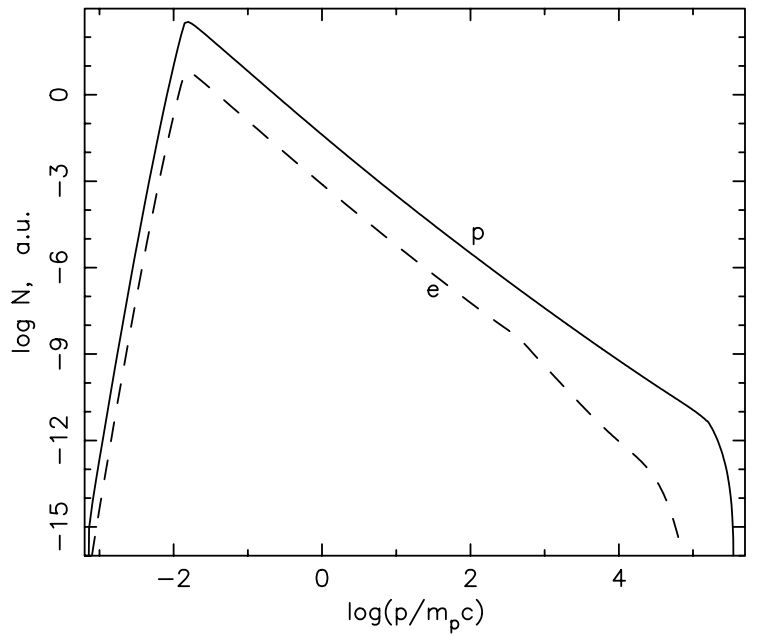

Fig. 2. The overall CR spectrum as function of momentum. Solid and dashed lines correspond to protons and electrons, respectively.

would not change at all for given radio flux. The magnetic field value which we deduce has a different character. We therefore defer its discussion to a later point of this section.

We now continue with the nonthermal aspects of Tycho's SNR.

Given the proton injection rate the acceleration process is characterized by a high efficiency in spherical symmetry: at the current time $t / t_{0}=0.7$ about $20 \%$ of the explosion energy have been already transferred to CRs, and the CR energy content $E_{\mathrm{c}}$ continues to increase to a maximum of about 43\% in the later Sedov phase (Fig. 1c), when particles start to leave the source. As usually predicted by the spherically symmetric model, such a CR acceleration efficiency is significantly higher than required for the average replenishment of the Galactic CRs by SNRs, corresponding to $E_{\mathrm{c}} \approx 0.1 E_{\mathrm{sn}}$. As discussed above (Völk et al. 2002; Berezhko et al. 2002), this requires a physical renormalization of the number of hadronic CRs. We choose $f_{\text {re }}=1 / 5$ here, in agreement with the theoretical estimate above, and consistent with the average Galactic CR replenishment.

With this renormalization the CRs inside Tycho's SNR contain at the current epoch the total energy

$E_{\mathrm{c}} \approx 0.2 f_{\mathrm{re}} E_{\mathrm{sn}} \approx 10^{49} \mathrm{erg}$.

The volume-integrated (or overall) CR spectrum

$N(p, t)=16 \pi^{2} p^{2} \int_{0}^{\infty} \mathrm{d} r r^{2} f(r, p, t)$

is given in Fig. 2 (Note that only the nonthermal part of the spectrum is pictured. That is why both the proton and electron spectrum have a maximum above suprathermal proton energies. As discussed above, the spectrum $N(p)$ is of course smoothly connected with the thermal distribution, not given here.) Far above suprathermal energies it has, for the case of protons, almost a pure power-law form $N \propto p^{-\gamma}$ over a wide momentum range from $10^{-2} m_{\mathrm{p}} c$ up to the cutoff momentum $p_{\max }=\epsilon_{\max } / c$, where $\epsilon_{\max } \approx 2 \times 10^{14} \mathrm{eV}$ is the maximum CR energy (Fig. 2). This value $p_{\max }$ is limited mainly by geometrical factors, which are the finite size and speed of the 
shock, its deceleration and the adiabatic cooling effect in the downstream region (Berezhko 1996). Due to the shock modification the power-law index slowly varies from $\gamma=2.2$ at $p \lesssim m_{\mathrm{p}} c$ to $\gamma=1.8$ at $p \gtrsim 10^{3} m_{\mathrm{p}} c$.

The shape of the overall electron spectrum $N_{\mathrm{e}}(p)$ deviates from that of the proton spectrum $N(p)$ at high momenta $p>$ $p_{1} \approx 10^{3} m_{\mathrm{p}} c$, due to the synchrotron losses in the downstream region with magnetic field $B_{\mathrm{d}} \approx 240 \mu \mathrm{G}$. This field is assumed to be roughly uniform and equal to the potshock field $B_{2}: B_{\mathrm{d}} \simeq$ $B_{2}=\sigma_{\mathrm{B}} B_{0}$, where $\sigma_{\mathrm{B}}^{2}=1 / 3+(2 / 3) \sigma^{2}$ for a strongly turbulent field. We shall approximately take $\sigma_{\mathrm{B}}=\sigma$ in the sequel.

The synchrotron losses become important for electron momenta greater than

$$
\frac{p_{1}}{m_{\mathrm{p}} c} \approx 1.3\left(\frac{10^{8} \mathrm{yr}}{t}\right)\left(\frac{10 \mu \mathrm{G}}{B_{\mathrm{d}}}\right)^{2} .
$$

Substituting the SN age $t=428$ yr into this expression, we have $p_{1} \approx 800 m_{\mathrm{p}} c$, in good agreement with the numerical results (Fig. 2).

The shock constantly produces the electron spectrum $f_{\mathrm{e}} \propto$ $p^{-q}$, with $q \approx 4$, up to the maximum momentum $p_{\max }^{\mathrm{e}}$ which is much larger than $p_{1}$. Therefore, within the momentum range $p_{1}$ to $p_{\max }^{\mathrm{e}}$, the electron spectrum is $f_{\mathrm{e}} \propto p^{-5}$ due to synchrotron losses, and the corresponding overall electron spectrum is $N_{\mathrm{e}} \propto p^{-3}$.

The maximum electron momentum can be estimated by equating the synchrotron loss time and the acceleration time, that gives

$$
\begin{aligned}
\frac{p_{\max }^{\mathrm{e}}}{m_{\mathrm{p}} c}= & 6.7 \times 10^{4}\left(\frac{V_{\mathrm{s}}}{10^{3} \mathrm{~km} \mathrm{~s}^{-1}}\right) \\
& \times \sqrt{\frac{(\sigma-1)}{\sigma\left(1+\sigma_{\mathrm{B}} \sigma\right)}\left(\frac{10 \mu \mathrm{G}}{B_{0}}\right)} .
\end{aligned}
$$

At the current epoch $V_{\mathrm{s}} \approx 3100 \mathrm{~km} \mathrm{~s}^{-1}$ which leads to a maximum electron momentum $p_{\max }^{\mathrm{e}} \approx 6 \times 10^{4} m_{\mathrm{p}} c$ in agreement with the numerical results (Fig. 2). Overall, the parameters $K_{\text {ep }}=4 \times 10^{-3}$ and $B_{\perp}=0.5 B_{\mathrm{d}}=120 \mu \mathrm{G}$ provide good agreement between the calculated and the measured synchrotron emission in the radio to X-ray ranges (Fig. 3). This concerns first the deviation of the radio-spectrum from the test particle form $S_{v} \propto v^{-0.5}$. At the same time, the steepening of electron spectrum at very high energies due to synchrotron losses naturally yields a fit to the X-ray data with their soft spectrum. Such a smooth spectral behavior is achieved in a $40 \mu \mathrm{G}$ upstream field (see also Berezhko et al. 2002). This is in clear contrast to the type of phenomenological fit by e.g. Reynolds (1998) for SN 1006.

Since the total number of accelerated protons has to be renormalized by the factor $f_{\mathrm{re}}=0.2$, the above number of accelerated electrons then corresponds to the renormalized parameter $K_{\text {ep }}=2 \times 10^{-2}$. This turns out to correspond reasonably well to the canonical value of $10^{-2}$ of the electron to proton ratio in the Galactic CRs. The total energy of accelerated electrons in Tycho's SNR is approximately $E_{\mathrm{c}}^{\mathrm{e}}=2 \times 10^{47} \mathrm{erg}$, as estimated from the fit to the observed synchrotron emission (Fig. 3) and our estimated value of $B_{\perp}$.

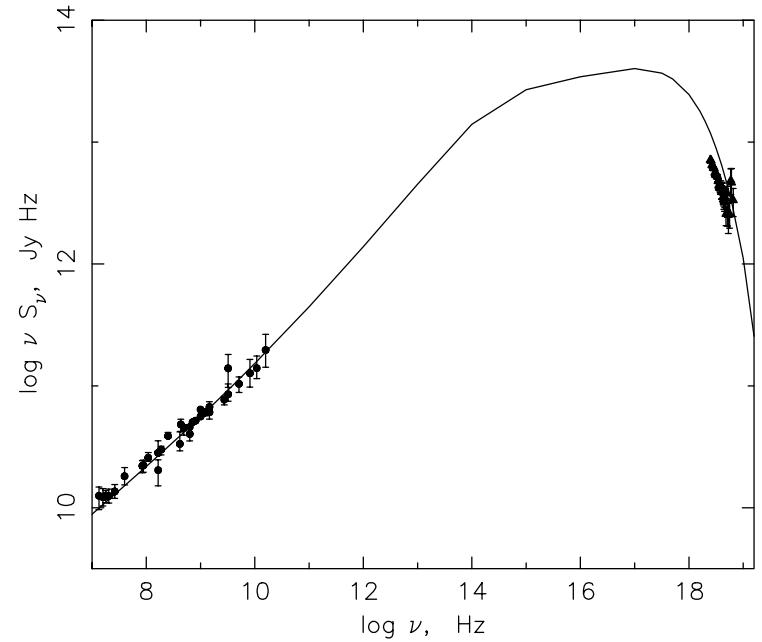

Fig. 3. Spectral energy distribution of the electron synchrotron emission. The observed X-ray (Allen et al. 1999) and radio emission (Reynolds \& Ellison 1992) are shown.

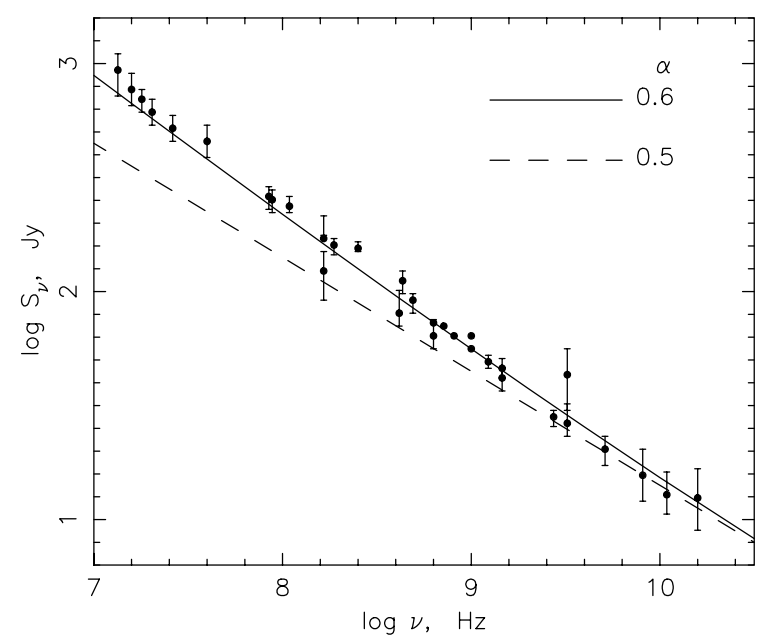

Fig. 4. Observed total radio flux of Tycho's SNR (Reynolds \& Ellison 1992) as a function of frequency, with model spectra superimposed. The solid curve represents our selfconsistent calculation, whereas the dashed curve illustrates the shape of the spectrum that would be expected in the test particle limit.

In detail the radio data can be fitted with a power law spectrum $S_{v} \propto v^{-\alpha}$, whose index $\alpha=0.607 \pm 0.007$ (Reynolds \& Ellison 1992) is considerably larger than 0.5 , as it would correspond to an electron spectrum $N_{\mathrm{e}} \propto p^{-2}$ produced in the test particle limit by an unmodified shock with compression ratio $\sigma=4$. For our choice of the proton injection rate, $\eta=3 \times 10^{-4}$, the shock is significantly modified by the backreaction of the accelerated protons (see Fig. 1b) with a total present compression ratio $\sigma=5.7$ at the present epoch. At the same time low energy electrons, with momenta $p \lesssim 10 m_{\mathrm{p}} c\left(\epsilon_{\mathrm{e}} \lesssim 10 \mathrm{GeV}\right)$ which produce synchrotron emission at $v \lesssim 10 \mathrm{GHz}$, are primarily accelerated at the subshock which only has a compression ratio $\sigma_{\mathrm{s}}=3.5$. Therefore these electrons have a steeper spectrum $N_{\mathrm{e}} \propto p^{-2.2}$ that leads to a radio spectrum $S_{v} \propto v^{-0.6}$ that fits the experimental data quite well (see Fig. 4, where the synchrotron flux at radio frequencies is presented). The fact 


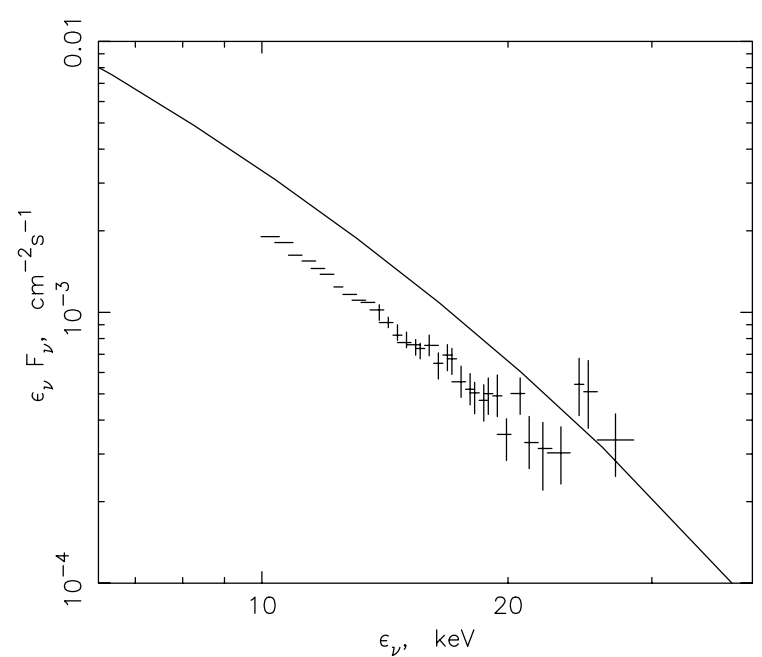

Fig. 5. Observed total integral hard X-ray flux of Tycho's SNR as a function of photon energy (Allen et al. 1999), together with the theoretical synchrotron spectrum.

that the observed value of the radio power law index $\alpha$ exceeds the value 0.5 is considered as an indication that the shock is significantly modified. Note that $\alpha=0.6$ is the average value of the power-law index within the frequency range shown in Fig. 4. In fact due to the concave shape of the electron spectrum the index slightly decreases with increasing frequency, with $\alpha=0.61$ and $\alpha=0.56$ at the lowest and largest frequency respectively. We emphasize that in our view not only a strongly modified shock, which produces a steep electron spectrum at energies $\epsilon_{\mathrm{e}}<1 \mathrm{GeV}$, but also a relatively high upstream magnetic field strength $B_{0}=40 \mu \mathrm{G}$, compared with typical ISM values $B_{0}=5 \mu \mathrm{G}$, and a corresponding downstream value $B_{\perp}=120 \mu \mathrm{G}$ are unavoidably required to have the energies of the radio emitting electrons in the steep part of their spectrum $\epsilon_{\mathrm{e}} \lesssim 1 \mathrm{GeV}$. According to the model calculations of Lucek \& Bell (2000), the existing ISM field can be significantly amplified near the shock by CR streaming to result in such values. An upper bound for the field strength is discussed below.

We note that the necessity of strong nonlinear shock modification and large values of the magnetic field strength in young SNRs to reproduce their steep radio spectra was argued for the first time by Reynolds \& Ellison (1992). The modified shock parameters and required magnetic field $B_{\mathrm{d}}=10^{-4}-10^{-3} \mathrm{G}$ which they derived for Tycho's SNR are consistent with our results.

The large perpendicular downstream magnetic field $B_{\perp}=$ $120 \mu \mathrm{G}$, which leads to a substantial steepening of the electron spectrum at high energies, also naturally provides a smooth cutoff in the synchrotron spectrum $S_{v}(v)$ at frequencies $v>$ $10^{18} \mathrm{~Hz}$, that correspond to X-ray photon energies $\epsilon_{v}>5 \mathrm{keV}$. As it can be seen from Fig. 5 the spectral shape of the calculated integral flux $\epsilon_{v} F_{v}\left(\epsilon_{v}\right)$ is closely similar to what is observed (Allen et al. 1999); the X-ray data are just approaching the cutoff region. The small difference in the amplitude is not relevant, because it could be easily reduced by a fine tuning of parameter values which we do not attempt to achieve here.

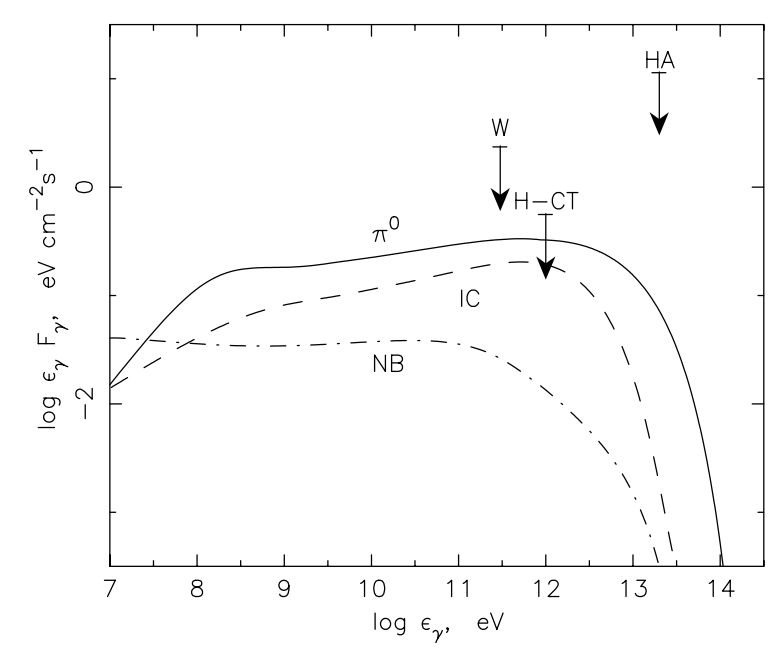

Fig. 6. IC (dashed line), NB(dash-dotted line), and $\pi^{0}$-decay (solid line) $\gamma$-ray spectral energy distributions, as a function of $\gamma$-ray energy. The observed $3 \sigma \gamma$-ray upper limits (W - Whipple, Buckley et al. 1998, H-CT - HEGRA IACT-system, Aharonian et al. 2001), and the 95\% confidence HA - HEGRA AIROBICC upper limit (Prahl et al. 1997), are shown as well.

The calculated IC emission off the CMB nonthermal bremsstrahlung (NB) and the derived $\pi^{0}$-decay $\gamma$-ray fluxes are presented in Fig. 6, together with existing experimental data. Note that the number of accelerated protons, which produce the $\pi^{0}$-decay $\gamma$-rays, is renormalized by the factor $f_{\text {re }}=0.2$ relative to the calculation in spherical symmetry.

With this renormalization, the hadronic gamma-ray flux is just below the HEGRA upper limit in Fig. 6. The electron synchrotron - and thus also IC and NB - fluxes have to remain the same, fixed by the radio and $\mathrm{X}$-ray observations.

According to the calculation, the hadronic $\gamma$-ray production exceeds the electron contribution by a factor of about 2 at energies $\epsilon_{\gamma} \sim 1 \mathrm{TeV}$, and dominates at $\epsilon_{\gamma}>10 \mathrm{TeV}$ (Fig. 6). The NB is always small above the $\mathrm{GeV}$ range. We cannot say much about lower energy NB because the electron distribution is not well known at those lower energies, as discussed earlier. In the region of the $\pi^{0}$-decay bump around $67.5 \mathrm{MeV}$, the hadronic gamma-ray spectrum may be distiguishable from the electron IC spectrum. However, the corresponding low particle energies are so far away from the "knee" in the Galactic CR spectrum around $10^{15} \mathrm{eV}$ that such a bump would not be a proof for Tycho's SNR being a typical source of the nuclear CRs. In addition, the steep background of the diffuse Galactic $\gamma$-ray flux would be hard to remove for an extended source, as most nearby SNRs would be.

The $\gamma$-ray spectra produced by the electronic and hadronic CR components have rather similar shapes at higher energies $10 \mathrm{GeV} \lesssim \epsilon_{\gamma} \lesssim 1 \mathrm{TeV}$ due to the synchrotron losses of the electrons. Therefore, the only clear observational possibility to discriminate between the leptonic and hadronic contributions is to measure the $\gamma$-ray spectrum at energies significantly higher than $1 \mathrm{TeV}$, where these two spectra are predicted to be essentially different. The detection of a substantial flux at energies $\epsilon_{\gamma} \gtrsim 10 \mathrm{TeV}$ would provide direct evidence for its hadronic origin. 


\subsection{B-field amplification possibilities; upper bound}

Strong shocks like those of young SNRs have their diffusive properties dominated by self-excited waves from the CR streaming instability (Bell 1978). In quasilinear approximation the mean square field fluctuation reaches a level $<\delta B^{2}>/ 4 \pi \simeq\left(V_{\mathrm{a}} / V_{\mathrm{s}}\right) 1 / 2 \rho V_{\mathrm{s}}^{2}$, where $1 / 2 \rho V_{\mathrm{s}}^{2}$ is the upstream flow energy density in the shock frame, and $V_{\mathrm{s}} / V_{\mathrm{a}} \gg 1$ is the Alfvénic Mach number of the shock, with $V_{\mathrm{a}}=B /(4 \pi \rho)^{1 / 2}$ (McKenzie \& Völk 1982, see also Drury 1983). Nevertheless $<\delta B^{2}>/ B^{2} \simeq V_{\mathrm{s}} / V_{\mathrm{a}}$ which implies that the rms wave field dominates the regular field.

Linear Alfvén waves constitute the long-wavelength elastic response of a magnetized plasma to a transverse perturbation. In reality it is not clear why the wave field could not grow even more strongly in the nonlinear regime due to a nonlinear evolution of the CR streaming instability. Such a "plastic" response (Völk \& McKenzie 1981) makes the distinction between a mean field and its fluctuations rather meaningless, effectively increasing the magnetic field in which particles undergo spatial diffusion (Völk 1984). Bell \& Lucek (2001) have constructed an interesting phenomenological model for such a nonlinear development, arguing for a strong reduction of the effective diffusion coefficient. For strong fluctuations the diffusion coefficient should come close to the Bohm limit $\kappa \sim 1 / 3 r_{\mathrm{g}} c$, with $r_{\mathrm{g}}=c p / e B$, where $B$ is the effective magnetic field strength. The maximum momentum to be reached in diffusive shock acceleration is then proportional to $B$ for any given time the process is assumed to operate.

How large the energy density of the effective field $B$ can actually become is a complex question. However, an upper bound is given by imposing no other constraint than the requirement that the Alfvén Mach number always remains large compared to unity, so that the shock continues to be strong and to produce the nonlinear field amplification in the first place. Requiring then $V_{\mathrm{s}} / V_{\mathrm{a}} \leq 5$ we obtain $B_{\mathrm{ub}}^{2} / 8 \pi=(1 / 25) 1 / 2 \rho V_{\mathrm{s}}^{2}$. Applying this to the shock of a type Ia SN, we take for the interstellar field $B^{\mathrm{ISM}}=5 \mu \mathrm{G}, V_{\mathrm{s}}=5 \times 10^{3} \mathrm{~km} \mathrm{~s}^{-1}$ during the sweep-up phase, and $B^{\mathrm{ISM}} /(4 \pi \rho)^{1 / 2} \simeq 14 \mathrm{~km} \mathrm{~s}^{-1}$, using our value of the ISM density ambient to Tycho's SNR. Thus $B_{\mathrm{ub}} / B^{\mathrm{ISM}} \simeq 71$ as upper bound for $B$.

Our estimate for the strength of $B$ in this paper consists in comparing the observed synchrotron spectrum with a theoretical spectrum for the shock accelerated electrons. We conclude that the $B$-value inferred is still well within this upper bound, especially considering that it is actually the mean square field value that counts.

An interesting corollary concerns the upper bound $p_{\mathrm{ub}}$ for the proton cutoff momentum which is achieved at the end of the sweep-up phase. Given that typical estimates yield $p_{\mathrm{ub}} \simeq$ $10^{14} \mathrm{eV}$ in SNRs for $B=B^{\mathrm{ISM}}$, we obtain for Tycho's SNR $p_{\mathrm{ub}} \simeq 7.1 \times 10^{15} \mathrm{eV}$ which corresponds to the so-called knee in the Galactic CR spectrum.

Even if we were to assume a much higher value $B^{\mathrm{ISM}} /(4 \pi \rho)^{1 / 2}=50 \mathrm{~km} \mathrm{~s}^{-1}$ for the ISM Alfvén velocity, these conclusions would not change qualitatively. This shows that at least in type Ia SNe the knee energy is the upper bound for diffusive shock acceleration.

\section{Summary}

The kinetic nonlinear model for CR acceleration in SNRs has been applied in detail to Tycho's SNR, in order to compare theoretical results with the recently found very low observational upper limit for the $\mathrm{TeV} \gamma$-ray flux. We have used stellar ejecta parameters $M_{\mathrm{ej}}=1.4 M_{\odot}, k=7$, distance $d=2.3 \mathrm{kpc}$, and ISM number density $N_{\mathrm{H}}=0.5 \mathrm{~cm}^{-3}$. A total hydrodynamic explosion energy $E_{\mathrm{sn}}=0.27 \times 10^{51}$ erg was derived to fit the observed size $R_{\mathrm{S}}$ and expansion speed $V_{\mathrm{s}}$ which are determined by the ratio $E_{\mathrm{sn}}^{2} / N_{\mathrm{H}}$. Even though the distance to the object is not very well known, the set of parameters has been shown to be internally consistent, and the predictions for the radio the and $\gamma$-ray fluxes are quite robust with respect to different parameter values in the literature.

A rather high downstream magnetic field strength $B_{\mathrm{d}} \sim$ $240 \mu \mathrm{G}$ and a proton injection rate $\eta=3 \times 10^{-4}$ are needed to reproduce the observed steep and concave radio spectrum and to ensure a smooth cutoff of the synchrotron emission in the X-ray region. We cannot exclude that the required magnetic field strength, that is significantly higher than the rms ISM value $5 \mu \mathrm{G}$, might have to be attributed in part to its nonlinear amplification near the SN shock by CR acceleration itself. The evidence for efficient nucleonic CR production that comes from the radio and X-ray data and leads to a strong shock modification, is even more definite for Tycho's SNR than in the case of SN 1006 (Berezhko et al. 2002).

We find that, after adjustment of the predictions of the nonlinear spherically-symmetric model by a physically necessary renormalization of the number of accelerated $\mathrm{CR}$ nuclei to take account of the quasi-perpendicular shock directions in a SNR, quite a reasonable consistency with most of the observational data can be achieved. The resulting nonthermal electron to proton ratio turns out to be consistent with the observed ratio in interstellar space. The total $\gamma$-ray flux at $1 \mathrm{TeV}$ (with the $\pi^{0}$-decay component exceeding the IC component) comes out to be slightly larger than the most restrictive observational upper limit from the HEGRA experiment. Given the remaining uncertainties in the basic astronomical parameters of Tycho, in particular those coming from analysis of the thermal $\mathrm{X}$-ray data, we do not consider this as a problem for the theory. It rather leads us to the prediction that detectors with several times higher sensitivity, like MAGIC or VERITAS in the Northern Hemisphere, should indeed detect this source above $100 \mathrm{GeV}$ in $\gamma$-rays.

The expected $\pi^{0}$-decay $\gamma$-ray flux $F_{\gamma}^{\pi} \propto \epsilon_{\gamma}^{-1}$ extends up to $\gtrsim 30 \mathrm{TeV}$, whereas the IC $\gamma$-ray flux has a cutoff above a few TeV. Therefore the detection of $\gamma$-ray emission at $\sim 10 \mathrm{TeV}$ would in addition imply clear evidence for a hadronic origin.

Acknowledgements. This work has been supported in part by the Russian Foundation for Basic Research (grants 00-02-17728, 99-02-16325). EGB and LTK acknowledge the hospitality of the Max-Planck-Institut für Kernphysik, where part of this work was carried out. GPR acknowledges the receipt of a von Humboldt fellowship.

\section{References}

Aharonian, F. A., Akhperjanian, A. G., Barrio, J. A., et al. 2001, A\&A, 373,292 
Allen, G. E., Gotthelf, E. V., \& Petre, R. 1999, in 26th ICRC, Salt Lake City, 3, 480

Aschenbach, B., Eggert, R., \& Trümper, J. 1995, Nature, 373, 587

Bell, A. R. 1978, MNRAS, 182, 147

Bell, A. R., \& Lucek, S. G. 2001, MNRAS, 321, 433

Berezhko, E. G. 1996, Astropart. Phys., 5, 367

Berezhko, E. G., \& Völk, H. J. 1997, Astropart. Phys., 7, 183

Berezhko, E. G., Elshin, V. K., \& Ksenofontov, L. T. 1996, JETP, 82, 1

Berezhko, E. G., Ksenofontov, L. T., \& Völk, H. J. 2002, A\&A, submitted [astro-ph/0204085]

Buckley, J. H., Akerlof, C. W., Carter-Lewis, D. A., et al. 1998, A\&A, 329,639

Chevalier, R. A. 1982, ApJ, 258, 790

Chevalier, R. A., Kirshner, R. P., \& Raymond, J. C. 1980, ApJ, 235, 186

Dickel, J. R., van Breugel, W. J. M., \& Strom, R. G. 1991, AJ, 101, 2151

Dickel, J. R., \& Jones, E. M. 1985, ApJ, 288, 707

Drury, L. O'C. 1983, Rep. Prog. Phys., 46, 973

Drury, L. O'C., Aharonian, F. A., \& Völk, H. J. 1994, A\&A, 287, 959

Hamilton, A. J. S., Sarazin, C. L., \& Symkowiak, A. E. 1986, ApJ, 300,713

Gull, S. F. 1973, MNRAS, 161, 47

Heavens, A. F. 1984, MNRAS, 211, 195
Jones, E. M., Smith, B. W., \& Straka, W. C. 1981, ApJ, 249, 185

Lucek, S. G., \& Bell, A. R. 2000, MNRAS, 314, 65

Malkov, M. A., \& Völk, H. J. 1995, A\&A, 300, 605

McKenzie, J. F., \& Völk, H. J. 1982, A\&A, 116, 191

Malkov, M. A., \& Drury, L. O’C. 2001, Rep. Prog. Phys., 64, 429

Prahl, J., \& Prosch, C. 1997, in 25th ICRC, Durban, 3, 217

Reynolds, S. P. 1998, ApJ, 493, 375

Reynolds, S. P., \& Ellison, D. C. 1992, ApJ, 399, L75

Reynolds, S. P., \& Gilmore, D. M. 1993, AJ, 106, 272

Reynoso, E. M., Moffett, D. A., Goss, W. M., et al. 1997, ApJ, 491, 816

Smith, A., Davelaar, J., Peacock, A., et al. 1988, ApJ, 325, 288

Strom, R. G., Goss, W. M., \& Shaver, P. A. 1982, MNRAS, 200, 473

Tan, S. M., \& Gull, S. F. 1985, MNRAS, 216, 949

Velázquez, P. F., Gómez, D. O., Dubner, G. M., et al. 1998, A\&A, 334, 1060

Völk, H. J. 1984, in High Energy Astrophysics, 19th Rencontres de Moriond, ed. Tran Than Van (Éditions Frontières, Gif sur Yvette), 281

Völk, H. J., \& McKenzie, J. F. 1981, Proc. 17th Int. Cosmic Ray Conf. (Paris), 9, 246

Völk, H. J., Berezhko, E. G., \& Ksenofontov, L. T. 2002, in preparation 